\title{
Spin-Dependent Ringing and Beats in a Quantum Dot System
}

\author{
Fabrício M. Souza \\ International Centre for Condensed Matter Physics, \\ Universidade de Brasília, 70904-910, Brasília-DF, Brazil
}

(Date: September 15, 2021)

\begin{abstract}
We report spin-dependent quantum coherent oscillations (ringing) and beats of the total and the spin currents flowing through a quantum dot with Zeeman split levels. The spin dependent transport is calculated via nonequilibrium Green function in the transient after a bias voltage is turned on at $t=0$. The dot is coupled to two electrodes that can be ferromagnetic or nonmagnetic. In the ferromagnetic case both parallel and antiparallel alignments are considered. The coherent oscillation and beat frequencies are controlled via the Zeeman energy $E_{Z}$. In particular, for $E_{Z}=0$ no beats are observed and the spin current is zero for nonmagnetic leads. In the ferromagnetic case a finite spin current is found for $E_{Z}=0$. The effects of temperature are also analyzed. We observe that with increasing temperature the ringing response and the beats tend to disappear. Additionally, the spin current goes to zero for nonmagnetic leads, remaining finite in the ferromagnetic case. The tunnel magnetoresistance (TMR) also reveals quantum coherent oscillations and beats, and it attains negative values for small enough temperatures and short times.
\end{abstract}

PACS numbers: PACS number

\section{INTRODUCTION}

Spin coherent dynamics and transport in quantum dots has attracted a lot of attention due to its relevance to the potential new generation of spintronic devices ${ }^{1}$ (e.g., Datta-Das transistor ${ }^{2}$ and storage devices $\left.{ }^{3.4 .5}\right)$, and for quantum computation and information processes,$\frac{6.7 .8}{1.8}$ where quantum coherence of the electron spin is desirable. Recent experiments demonstrate the possibility to coherently manipulate quantum states of single and double electron spins in quantum dot systems $9,10,11,12,13,14,15,16$ Such control can be achieved, for instance, via fast bias/gate voltage pulses, electron spin resonance (ESR) fields and coherent optical fields. Those experiments indicate the feasibility of using a single electron in quantum dot system as a quantum bit and reveal encouraging spin coherence lifetimes for quantum processing.

In addition, a variety of interesting spin-coherent effects have been reported during recent years. For instance, coherent Rabi oscillations generated by an ESR field were studied both theoretically 17 and experimentally 13 in quantum dot systems in the Coulomb blockade regime. Coherent spin-dynamics was probed via time-resolved Faraday rotation (TRFR) technique, revealing coherent dynamics of net carrier spins in semiconductor bulk, quantum well and quantum dots $\underline{18,19,20}$ In addition to quantum coherent oscillations, quantum beats of the spin magnetization due to Zeeman splitting in a semiconductor quantum dot system were observed via time-resolved Faraday rotation technique, 19 and quantum beats in the TRFR signal were measured for magnetic fields higher than $5 \mathrm{~T}$ in a quantum dot system $\stackrel{14}{ }$ Quantum coherent beating in the magnetoresistance of a double-barrier structure with both diluted magnetic semiconductor (DMS) well and contacts was

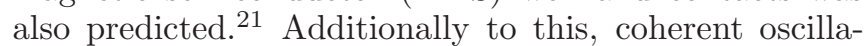

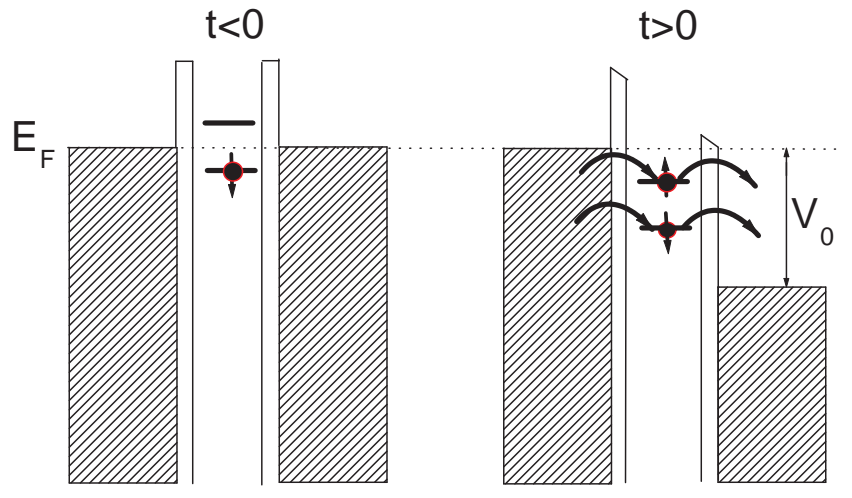

FIG. 1: System studied: a quantum dot attached to two leads via tunnel barriers. Both ferromagnetic and nonmagnetic leads are considered. The dot level is Zeeman-split due to a external magnetic field. Before the bias voltage is turned on $(t<0)$ the spin split level $\epsilon_{\downarrow}$ is energetic accessible for the electrons in the leads, while $\epsilon_{\uparrow}\left(>E_{F}\right)$ is forbidden. After a bias voltage $V_{0}$ is applied $(t>0)$ both levels become inside the conduction window and a spin-dependent tunnel current arises.

tions of polarized current in magnetotransport through Zeeman split levels of quantum dots were investigated via quantum rate equations ${ }^{22}$

Quantum coherent oscillations (ringing) of the current flowing through a single level quantum dot attached to nonmagnetic leads was predicted in a transient time scale after a bias voltage is turned on: $23,24,25,26$ Here we describe transport in the same transient regime but including spin-dependent effects. An understanding of the spinfull case can better guide possible experimental measurements of the ringing response which now comes up with new spin-based signatures. Here we apply the nonequilibrium Green function (NEGF) formulation developed in 24] to account for (i) Zeeman splitting of the dot level 
and/or (ii) ferromagnetic leads. These two additional features introduce new spin-dependent effects. For example, the coherent oscillations reported previously in [23, 24 25 become spin-dependent with slightly different frequencies for each spin component of the current. This spin-splitting of the frequency generates quantum coherent beats of the total current $I=I_{\uparrow}+I_{\downarrow}$ and of the spin current $I^{s}=I_{\uparrow}-I_{\downarrow}$. We also find a dynamical tunnel magnetoresistance (TMR) that shows coherent oscillations, coherent beats and attains negative values in the transient regime for small enough temperatures.

The paper is organized as follows: in Sec. II we describe the system and the formulation adopted, in Sec. III we present and discuss the results and in Sec. IV we conclude.

\section{MODEL AND FORMULATION}

Figure 1 illustrates the system considered. It is composed of one quantum dot coupled via tunnel barriers to a left $(\mathrm{L})$ and to a right $(\mathrm{R})$ lead. Both ferromagnetic and nonmagnetic leads are considered. For the ferromagnetic case both parallel (P) and antiparallel (AP) alignments are analyzed. We assume that for zero bias voltage $(t<0)$ the spin- $\uparrow$ and spin- $\downarrow$ levels are above and below the Fermi energy $E_{F}$ of the reservoirs, respectively. This prepared configuration gives rise to a relatively high transient spin current in the case of nonmagnetic leads as we will describe in Sec. III(a). When the bias voltage is turned on $(t>0)$ both levels attain resonance with the emitter states, thus resulting in the subsequent spin dynamics.

The total Hamiltonian of the system is given by $H=$ $H_{L}+H_{R}+H_{D}+H_{T}$, where

$$
H_{L / R}=\sum_{\mathbf{k} \sigma} \epsilon_{\mathbf{k} \sigma L / R}(t) c_{\mathbf{k} \sigma L / R}^{\dagger} c_{\mathbf{k} \sigma L / R},
$$

with $\epsilon_{\mathbf{k} \sigma L / R}(t)=\epsilon_{\mathbf{k} \sigma L / R}^{0}+\Delta_{L / R}(t)$ being the freeelectron energy with a time-dependent contribution $\Delta_{L / R}(t)$ due to the bias voltage. The operator $c_{\mathbf{k} \sigma L / R}$ $\left(c_{\mathbf{k} \sigma L / R}^{\dagger}\right)$ annihilates (creates) one electron with wave vector $\mathbf{k}$ and spin $\sigma$ in the left $(L)$ or right $(R)$ lead. For the dot Hamiltonian we have

$$
H_{D}=\sum_{\sigma}\left[\epsilon_{d}(t)+\sigma E_{Z} / 2\right] d_{\sigma}^{\dagger} d_{\sigma},
$$

where $\epsilon_{d}(t)=\epsilon_{0}+\Delta_{d}(t)$ is the dot level with a timedependent term $\Delta_{d}(t), E_{Z}$ is the Zeeman energy due to some external magnetic field 27 and $\sigma=+$ or - for spin $\uparrow$ or $\downarrow$, respectively. We neglect the Zeeman splitting of the leads $E_{Z}^{\text {leads }}$ in our model $\underline{28}$ The operators $d_{\sigma}$ and $d_{\sigma}^{\dagger}$ annihilate and create, respectively, one electron with spin $\sigma$ in the $\operatorname{dot} \stackrel{29}{ }$ For the leads-dot coupling we have

$$
H_{T}=\sum_{\mathbf{k} \sigma \eta}\left(V c_{\mathbf{k} \eta}^{\dagger} d_{\sigma}+V^{*} d_{\sigma}^{\dagger} c_{\mathbf{k} \sigma \eta}\right),
$$

where $V$ is a constant coupling parameter. We do not account for spin relaxation and spin decoherence in our model, which is reasonable for short enough times. Previous studies have found for the relaxation and decoherence times, $T_{1}$ and $T_{2}$ respectively, typically $T_{1} \sim 1-20$ $\mathrm{ms}^{5,10,30,31}$ and $T_{2} \gtrsim \mu \mathrm{s}, \underline{11,32,33}$

The time dependent current is given by a sum of the currents flowing into and out of the dot, $I_{\sigma}^{\eta}(t)=I_{\sigma}^{\eta, i n}(t)+$ $I_{\sigma}^{\eta, \text { out }}(t)$. In the noninteracting case and wideband limit they read ${ }^{34}$

$$
I_{\sigma}^{\eta, i n}(t)=-\frac{e \Gamma_{\sigma}^{\eta}}{\hbar} \int \frac{d \epsilon}{\pi} f_{\eta}(\epsilon) \operatorname{Im}\left[A_{\sigma \eta}(\epsilon, t)\right],
$$

and

$$
I_{\sigma}^{\eta, \text { out }}(t)=-\frac{e \Gamma_{\sigma}^{\eta}}{\hbar} \int \frac{d \epsilon}{2 \pi} \sum_{\xi=L, R} \Gamma_{\sigma}^{\xi} f_{\xi}(\epsilon)\left|A_{\sigma \xi}(\epsilon, t)\right|^{2},
$$

where $\Gamma_{\sigma}^{\eta}$ is the tunneling rate, $\Gamma_{\sigma}^{\eta}=2 \pi|V|^{2} \rho_{\sigma}^{\eta}$, with $\rho_{\sigma}^{\eta}$ being the constant density of states for spin $\sigma$ in lead $\eta(=L, R)$. The ferromagnetism of the leads is accounted for via the model $\Gamma_{\sigma}^{L}=\Gamma_{0}(1+\sigma p)$ and $\Gamma_{\sigma}^{R}=\Gamma_{0}(1 \pm \sigma p)$, where $\Gamma_{0}$ is the leads-dot coupling strength and $p$ gives the polarization degree of the leads. The signs +/in $\Gamma_{\sigma}^{R}$ apply for parallel and antiparallel configurations, respectively $\underline{\underline{35}}$ The function $f_{\eta}(\epsilon)$ is the Fermi distribution function for lead $\eta$. For a bias voltage of the kind $\Delta_{L}=0, \Delta_{R}=-V_{0} \theta(t)$ and $\Delta_{d}=\Delta_{R} / 2$ the function $A_{\sigma \eta}(\epsilon, t)$ is given by $\underline{36}$

$$
\begin{gathered}
A_{\sigma \eta}(\epsilon, t>0)=\frac{e^{i\left[\epsilon-\epsilon_{0}-\sigma E_{Z} / 2-\Delta_{d}+\Delta_{\eta}+i\left(\Gamma_{\sigma}^{L}+\Gamma_{\sigma}^{R}\right) / 2\right] t / \hbar}}{\epsilon-\epsilon_{0}-\sigma E_{Z} / 2+i\left(\Gamma_{\sigma}^{L}+\Gamma_{\sigma}^{R}\right) / 2}+ \\
\frac{1-e^{i\left[\epsilon-\epsilon_{0}-\sigma E_{Z} / 2-\Delta_{d}+\Delta_{\eta}+i\left(\Gamma_{\sigma}^{L}+\Gamma_{\sigma}^{R}\right) / 2\right] t / \hbar}}{\epsilon-\epsilon_{0}-\sigma E_{Z} / 2-\Delta_{d}+\Delta_{\eta}+i\left(\Gamma_{\sigma}^{L}+\Gamma_{\sigma}^{R}\right) / 2}
\end{gathered}
$$

Using this expression inside Eqs. (4) and (5) we find the results presented in the next section.

For comparison we apply the master equation (ME) technique to calculate the current and spin-current. While the NEGF is valid for both $k_{B} T \gg \Gamma_{0}$ and $k_{B} T \ll$ $\Gamma_{0}$, the ME approach is accurate only for $k_{B} T \gg \Gamma_{0}$ (sequential-tunneling limit). So we will restrict the comparison only for relatively high temperatures, which correspond to the range of mutual validity of the two approaches. The current expression in the ME formulation is given by $\underline{37}$

$$
I_{\sigma}^{\eta}=e \Gamma_{\sigma}^{\eta}\left[f_{\eta \sigma} P_{0}-\left(1-f_{\eta \sigma}\right) P_{\sigma}+\tilde{f}_{\eta \sigma} P_{\bar{\sigma}}-\left(1-\tilde{f}_{\eta \sigma}\right) P_{2}\right],
$$

where $P_{0}, P_{\sigma}$ and $P_{2}$ are the probabilities to have no electron, one electron with spin $\sigma$ and two electrons, respectively, in the dot. The Fermi functions are $f_{\eta \sigma}=$ $\left\{1+\exp \left[\left(\epsilon_{\sigma}-\Delta_{\eta}\right) /\left(k_{B} T\right)\right]\right\}^{-1}$ and $\tilde{f}_{\eta \sigma}=\left\{1+\exp \left[\left(\epsilon_{\sigma}+U-\right.\right.\right.$ $\left.\left.\left.\Delta_{\eta}\right) /\left(k_{B} T\right)\right]\right\}^{-1}$ where $\epsilon_{\sigma}=\epsilon_{d}+\sigma E_{Z} / 2$. Defining the vector of the occupation probabilities $\underline{\mathbf{P}}=\left(P_{0}, P_{\uparrow}, P_{\downarrow}, P_{2}\right)^{T}$ we can write the master equation as

$$
\underline{\dot{\mathrm{P}}}=\underline{\underline{\mathrm{M}}} \underline{\underline{\mathrm{P}}},
$$

where the transition matrix is given by $\underline{\underline{\mathbf{M}}}=\underline{\underline{\mathrm{M}}}^{\mathbf{L}}+\underline{\underline{\mathbf{M}}}^{\mathbf{R}}$, with 


$$
\underline{\underline{\underline{\mathbf{M}}}}^{\eta}=\left(\begin{array}{cccc}
-\Gamma_{\uparrow}^{\eta} f_{\eta \uparrow}-\Gamma_{\downarrow}^{\eta} f_{\eta \downarrow} & \Gamma_{\uparrow}^{\eta}\left(1-f_{\eta \uparrow}\right) & \Gamma_{\downarrow}^{\eta}\left(1-f_{\eta \downarrow}\right) & 0 \\
\Gamma_{\uparrow}^{\eta} f_{\eta \uparrow} & -\Gamma_{\uparrow}^{\eta}\left(1-f_{\eta \uparrow}\right)-\Gamma_{\downarrow}^{\eta} \tilde{f}_{\eta \downarrow} & 0 & \Gamma_{\downarrow}^{\eta}\left(1-\tilde{f}_{\eta \downarrow}\right) \\
\Gamma_{\downarrow}^{\eta} f_{\eta \downarrow} & 0 & -\Gamma_{\uparrow}^{\eta} \tilde{f}_{\eta \uparrow}-\Gamma_{\downarrow \downarrow}^{\eta}\left(1-f_{\eta \downarrow}\right) & \Gamma_{\uparrow}^{\eta}\left(1-\tilde{f}_{\eta \uparrow}\right) \\
0 & \Gamma_{\downarrow}^{\eta} \tilde{f}_{\eta \downarrow} & \Gamma_{\uparrow}^{\eta} \tilde{f}_{\eta \uparrow} & \Gamma_{\uparrow}^{\eta} \tilde{f}_{\eta \uparrow}+\Gamma_{\downarrow}^{\eta} \tilde{f}_{\eta \downarrow}-\left(\Gamma_{\uparrow}^{\eta}+\Gamma_{\downarrow}^{\eta}\right)
\end{array}\right) .
$$

If we take $f_{R \sigma}=\tilde{f}_{R \sigma}=0$ we obtain a matrix form for $\mathbf{M}$ similar to the one presented in 38]. Even though Eqs. (7)-(9) can account for Coulomb interaction in the sequential limit, we are interested in comparing the results obtained from both the ME and the NEGF (which is noninteracting in the present formulation), thus we simply assume $U=0$ in the above ME expressions. Some effects of Coulomb interaction in a transient response (sequential limit) can be found, for instance, in [39].

\section{RESULTS}

\section{A. Nonmagnetic Leads}

Figure 2 shows the time evolution of the total current $I=I_{\uparrow}+I_{\downarrow}$ and the spin current $I^{s}=I_{\uparrow}-I_{\downarrow}$ in the emitter lead, $\stackrel{40}{=}$ obtained via NEGF. Different Zeeman energies are considered. For $E_{Z}=0$ there is no spin current [Fig. 2(b)] and the current [Fig. 2(a)] presents the typical coherent oscillations that arise after a bias voltage is turned on $23,24,25,26$ The oscillatory frequency is given by $\hbar \omega_{c}=\left|E_{F}-\epsilon_{d}\right|$ (or equivalently the period $\left.T_{c}=2 \pi \hbar /\left|E_{F}-\epsilon_{d}\right|\right)$ and the damping is due to the leads-dot coupling. We may note that for the parameters adopted in Fig. 2(a) we have $T_{c} \approx 0.06 \hbar / \Gamma_{0} \underline{\underline{41}} \mathrm{In}$ the presence of Zeeman splitting the frequency $\omega_{c}$ becomes spin dependent, with $\hbar \omega_{c}^{\sigma}=\left|E_{F}-\epsilon_{d}-\sigma E_{Z} / 2\right|$, so the spin components of the current oscillate with distinct frequencies. In the case of relatively close frequencies, quantum beats of $I$ and $I^{s}$ are seen, with beating frequency given by $\hbar\left|\omega_{c}^{\uparrow}-\omega_{c}^{\downarrow}\right|=E_{Z}$ [Figs. 2(c)-(f)].

The spin current observed in the presence of Zeeman energy [Figs. 2(d) and (f)] comes from the initial configuration $(t<0)$ of the system. For $t<0$ the occupations are $n_{\downarrow} \approx 1$ and $n_{\uparrow} \approx 0$. When the bias voltage is turned on at $t=0$ the spin $\uparrow$ electrons in the emitter lead can start to flow through the dot, while the spin $\downarrow$ ones have to wait till the initial $\downarrow$ electron in the dot leaves to the collector lead (Pauli blockade). Since this tunnel event takes a typical time of $\hbar / \Gamma_{0}$ a spin current is expected in this time range. In the case of initially both $n_{\uparrow} \approx 0$ and $n_{\downarrow} \approx 0$, which can be achieved by taking $\epsilon_{0}$ high enough in order to forbid thermal occupation of the spin split levels, an oscillatory spin current around zero is observed.

In Fig. (3) we show $I_{\uparrow}$ and $I_{\downarrow}$ separately. For $E_{Z}=$ 0 the currents $I_{\uparrow}$ and $I_{\downarrow}$ coincide, while for $E_{Z}=10$ and $E_{Z}=20$ they differ, thus generating spin current.

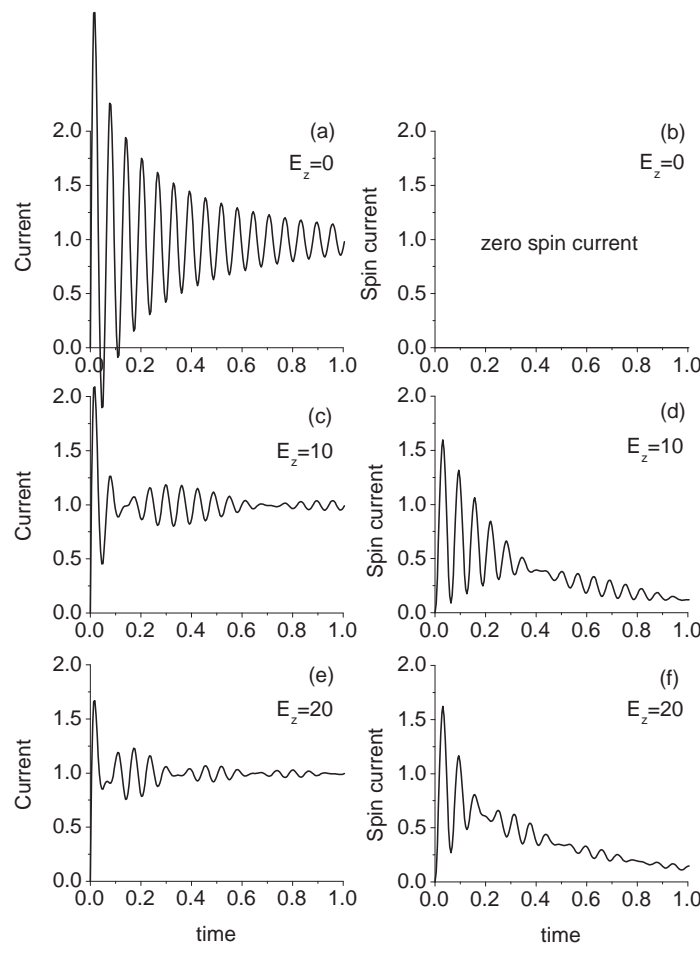

FIG. 2: Total current $I_{\uparrow}+I_{\downarrow}$ (left panels) and spin current $I_{\uparrow}-I_{\downarrow}$ (right panels) against time for different Zeeman energies $E_{Z}$. For $E_{Z}=0$ the current shows coherent oscillations (ringing) with a period $T_{c}=2 \pi \hbar /\left|E_{F}-\epsilon_{d}\right|$. No spin current is observed for $E_{Z}=0$. In contrast, for $E_{Z}=10$ and $E_{Z}=20$ a spin current arises and quantum coherent beats are seen in both current and spin current. Units: $e \Gamma_{0} / \hbar$ for the currents, $\hbar / \Gamma_{0}$ for the time and $\Gamma_{0}$ for the energies. Parameters: $\epsilon_{0}=0$, $V_{0}=200, k_{B} T=0.1, p=0$.

Eventually for high enough times $(t \gg 1)$ both $I_{\uparrow}$ and $I_{\downarrow}$ become close to each other, thus resulting in $I^{s} \rightarrow 0$. The difference in frequency for each spin component is clearly seen in Figs. 3(b)-(c). In particular as $E_{Z}$ increases, we observe a blue shift for the frequency $\omega_{c}^{\downarrow}$ and a red shift for $\omega_{c}^{\uparrow}$.

In Fig. (44) we analyze the effects of temperature on the coherent oscillations. We find that as $k_{B} T$ increases the oscillatory behavior tends to disappear in both the current and the spin-current. This is expected since with increasing $T$ the coherence is washed out. For comparison we show in Fig. [4(e)-(f) the total and the spin currents calculated via the master equation technique (dots). We 


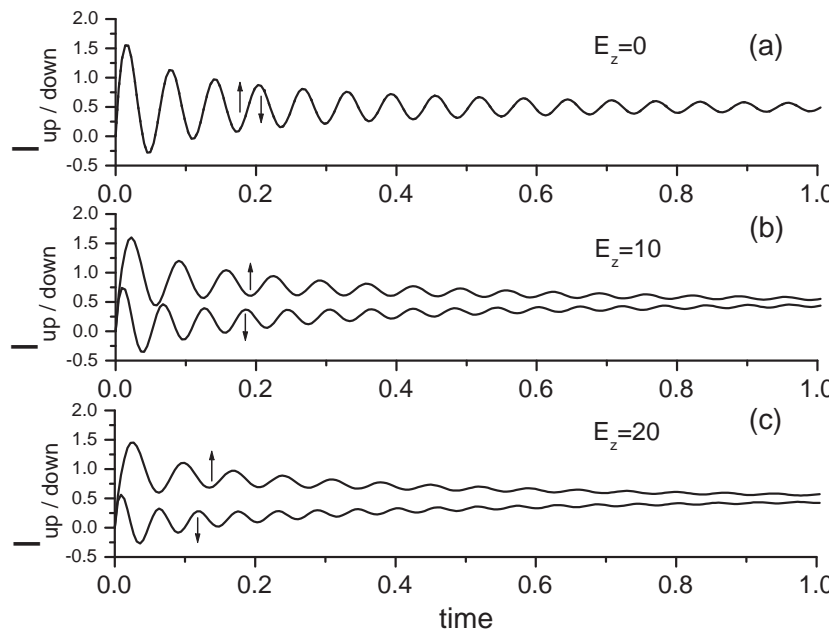

FIG. 3: Spin resolved currents (denoted by up and down arrows close to the curves) against time for three different Zeeman energies. For $E_{Z}=0, I_{\uparrow}$ and $I_{\downarrow}$ coincide. As $E_{Z}$ increases we find a red-shift for the frequency of $I_{\uparrow}$ and a blueshift for the frequency of $I_{\downarrow}$. This frequency splitting results in the quantum beats seen in Fig. 2. Units and parameters as in Fig. 2.

find close agreement between the nonequilibrium Green function and the master equation results, except by the strongly suppressed coherent oscillations still seen in the NEGF calculation for $k_{B} T=10$. As the temperature increases these coherent oscillations (ringing) disappear and both NEGF and ME approaches give equal results. Additionally to this, we note that the spin current is suppressed with increasing $k_{B} T$. For even higher temperatures we find zero spin current for all times, which is related to the fact that $n_{\uparrow}(t<0)$ approaches $n_{\downarrow}(t<0)$ with increasing $k_{B} T$-thus resulting in a less effective transient Pauli blockade. When the leads are ferromagnetic, though, the source of spin current is not only the initial configuration of the system but also the leads itself, so $I^{s}$ remains nonzero even for high temperatures, as we describe next.

\section{B. Ferromagnetic Leads}

Figure (5) shows the current and the spin current against time in the presence of ferromagnetic leads for differing temperatures and $E_{Z}=10$. Both $\mathrm{P}$ and $\mathrm{AP}$ configurations are analyzed. Here we have enlarged the time scale compared to Fig. 4 because some contrasting features between $\mathrm{P}$ and $\mathrm{AP}$ alignments are better seen after $t=1$ (e.g., $\left.I_{P}>I_{A P}\right)$. For $t \lesssim 1$ the standard inequality $I_{P}>I_{A P}$ is only true when we approach the sequential limit for $k_{B} T=10$. Curiously, for $k_{B} T=0.1$ and $k_{B} T=1$ we find $I_{A P}$ slightly above $I_{P}$ for $t \lesssim 1$. This unusual behavior can be qualitatively understood in terms of tunnel rates and the initial (prepared) spin-
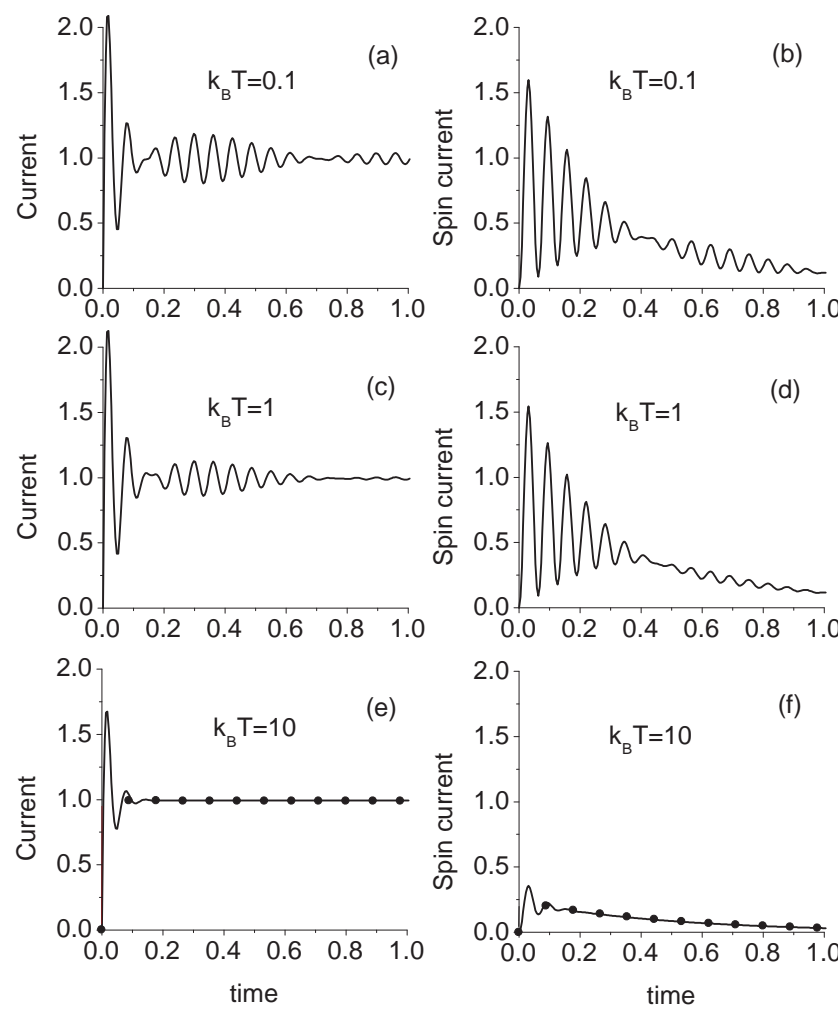

FIG. 4: Total current (left panels) and spin current (right panels) against time for differing temperatures. Quantum coherent oscillations and beats are seen for $k_{B} T=0.1$ and $k_{B} T=1$. As the temperature increases the oscillations vanish (see $\left.k_{B} T=10\right)$. Additionally, the spin current is significantly suppressed for $k_{B} T=10$. For comparison in panels (e) and (f) we show the results obtained via master equation (dots). Units as in Fig. 2. Parameters: $\epsilon_{0}=0, V_{0}=200, E_{Z}=10$, $p=0$.

population of the dot. Initially $(t<0)$ the dot is occupied by one spin $\downarrow$ electron. When the bias voltage is applied $(t>0)$ this electron can leave the dot to the collector lead with a tunnel rate given by $\Gamma_{\downarrow}^{R}$. Since $\Gamma_{\downarrow}^{R}$ is bigger for AP than for $\mathrm{P}$ configuration, the initial spin $\downarrow$ electron leaves the dot faster in the AP alignment. This turns the Pauli blockade less effective in this configuration, thus allowing the emitter current $I_{A P}$ to be slightly greater than $I_{P}$ at initial times.

When $k_{B} T$ exceeds the coupling strength $\Gamma_{0}$, the coherent oscillations of both the current and the spin current are strongly suppressed and the standard inequality $I_{P}>I_{A P}$ is recovered even for short times after $t=0$. Here we also see an agreement between the results obtained via NEGF and ME, except by the coherent oscillations residually present in the NEGF calculation for $k_{B} T=10$. As the temperature increases even further these residual oscillations of the current and the spincurrent are washed out and both NEGF and ME approaches converge to the same results. Finally, we observe that the spin current does not tend to zero with in- 

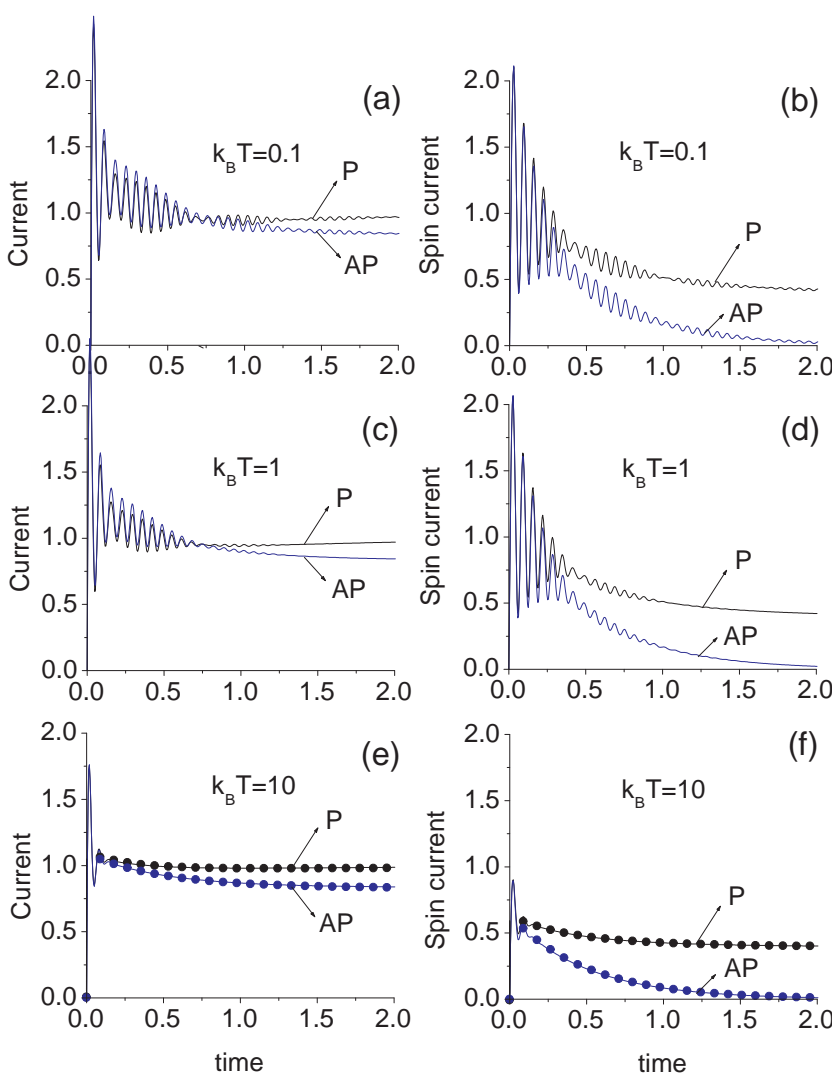

)

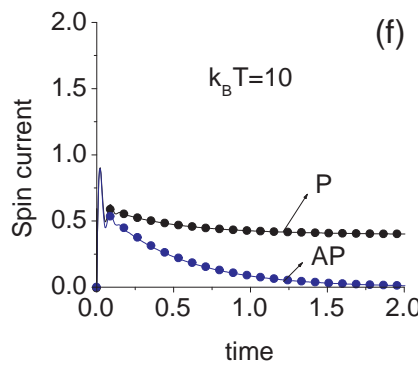

FIG. 5: (Color online) Total current $I=I_{\uparrow}+I_{\downarrow}$ (left panels) and spin current $I^{s}=I_{\uparrow}-I_{\downarrow}$ (right panels) against time for differing temperatures in the case of ferromagnetic leads. Both parallel (P) and antiparallel (AP) configurations are shown. Coherent oscillations and beats are seen in both alignments. The standard inequality $I_{P}>I_{A P}$ (magnetoresistance effect) is seen for $t \gtrsim 1$ and $k_{B} T=0.1$ or $k_{B} T=1$. For times $t \lesssim 1$ unusual behaviors like $I_{P}<I_{A P}$ can be found for these temperatures. In contrast, for $k_{B} T=10$ almost no oscillations are seen and $I_{P}>I_{A P}$ is recovered for all times. For comparison in panels (e)-(f) we show results obtained via master equation technique (dots). We also note that the spin current goes to zero as the time evolves in the AP alignment while remains finite in the $\mathrm{P}$ case even for $k_{B} T=10$. Units as in Fig. 2 Parameters: $\epsilon_{0}=0, V_{0}=200, E_{Z}=10, p=0.4$.

creasing temperature as we see in the nonmagnetic case [compare Figs. 4(f) and $5(\mathrm{f})]$. The suppression of $I^{s}$ is observed only with increasing time in the AP alignment, remaining finite for the stationary limit in the $\mathrm{P}$ configuration.

In Fig. (6) we show the time-dependent TMR, defined as $\mathrm{TMR}=\left(I_{P}-I_{A P}\right) / I_{A P}$. Coherent oscillations and quantum beats are seen for both $k_{B} T=0.1$ and 1 and is only residually observed for $k_{B} T=10$. Interestingly, the TMR attains negative values for short times and small temperatures $\underline{\underline{42}}$ In panel (c) we compare the TMR obtained via NEGF (solid line) and ME (dots) with essentially no contrast between them.

Finally in Fig. 7 we show the spin resolved cur-
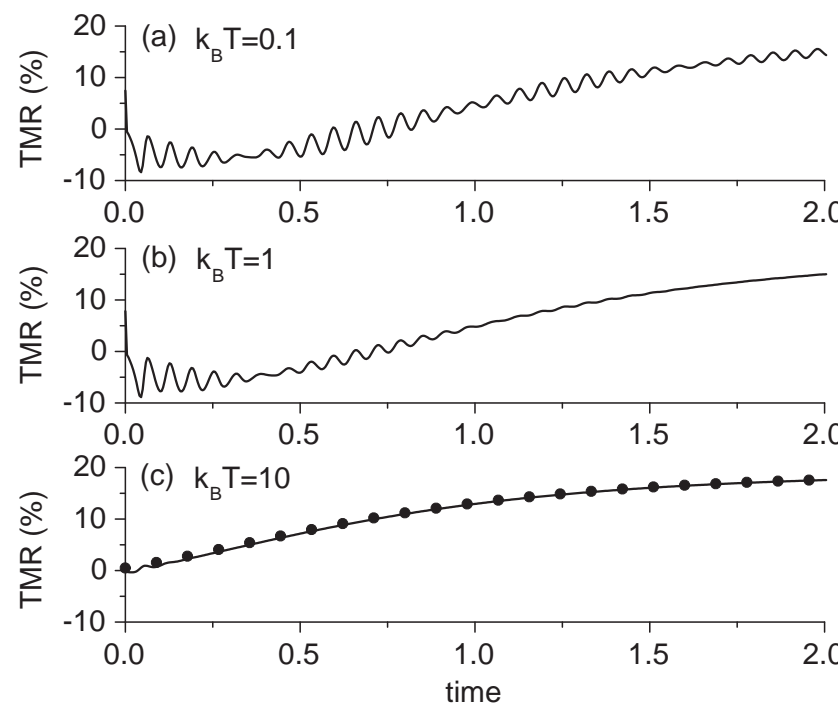

FIG. 6: Tunnel magnetoresistance (TMR) against time for increasing $k_{B} T$. For $k_{B} T=0.1$ and 1 the TMR shows a ringing response, quantum beats and attains negative values before it increases to a stationary value close to $20 \%$. In contrast, for $k_{B} T=10$ the TMR is always positive with almost no oscillations. The dots show the TMR obtained via the master equation technique. Units as in Fig. 2. Parameters: $\epsilon_{0}=0$, $V_{0}=200, E_{Z}=10, p=0.4$.

rents and the spin current in the ferromagnetic case for $E_{Z}=0$. Here $I^{s} \neq 0$ [in contrast to the nonmagnetic case, Fig. 2(b)] and no beats are seen since $I_{\uparrow}$ and $I_{\downarrow}$ have the same frequency. Additionally, the oscillation amplitudes and the decaying rates can differ for each spin component. In the parallel configuration we observe that $I_{\uparrow}$ starts oscillating with a higher amplitude than $I_{\downarrow}$ but its oscillations are faster suppressed. This is due to the inequality $\Gamma_{\uparrow}^{L}+\Gamma_{\uparrow}^{R}>\Gamma_{\downarrow}^{L}+\Gamma_{\downarrow}^{R}$. The interplay between oscillation amplitudes and decaying rates gives rise to a node in the spin current seen around $t=0.5$ [Fig. 7(b)]. For long enough times both $I_{\uparrow}$ and $I_{\downarrow}$ attain their respective stationary values with $I_{\uparrow}>I_{\downarrow}$ [Fig. 7(a)]. In the AP alignment $I_{\uparrow}$ starts oscillating with a higher amplitude than $I_{\downarrow}$ but, in contrast to the $\mathrm{P}$ case, the decaying rate is the same for both spin component $\left(\Gamma_{\uparrow}^{L}+\Gamma_{\uparrow}^{R}=\Gamma_{\downarrow}^{L}+\Gamma_{\downarrow}^{R}\right)$. Consequently no node is observed in the spin current, which simply oscillates decaying to zero.

\section{CONCLUSION}

We have calculated spin-dependent transport through a Zeeman split quantum dot level after a bias voltage is turned on. The dot is coupled to two leads (source and drain) that can be ferromagnetic or nonmagnetic. Quantum coherent oscillations (ringing) and beats of the current and of the spin current are found in a transient 

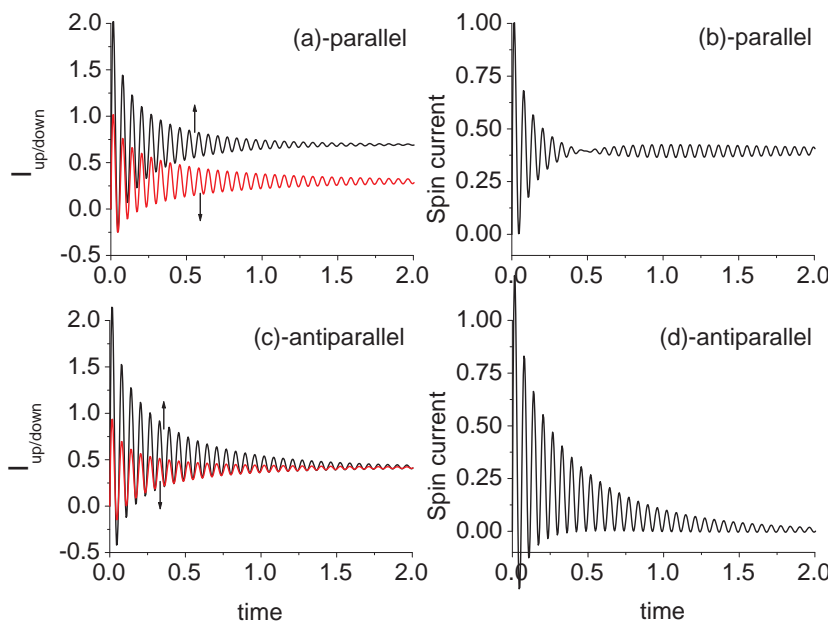

FIG. 7: (Color online) Spin resolved currents (denoted by up and down arrows closed to the curves) and spin current for both $\mathrm{P}$ and $\mathrm{AP}$ alignments and $E_{Z}=0$. Both $I_{\uparrow}$ and $I_{\downarrow}$ oscillate with the same frequency due to $E_{Z}=0$, but in contrast to the nonmagnetic case [Fig. 3(a)] here the amplitude and decaying rate $\left(\Gamma_{\sigma}^{L}+\Gamma_{\sigma}^{R}\right)$ can differ for each spin component. In particular in the $\mathrm{P}$ alignment the interplay between the amplitudes and the decaying rates gives rise to a node in the spin current. Units as in Fig. 2 Parameters: $\epsilon_{0}=0$, $V_{0}=200, E_{Z}=0, k_{B} T=0.1, p=0.4$. time scale. The frequency of the beats can be tuned via the Zeeman splitting energy $E_{Z}$. We also report a spin current $I^{s}$ that arises when $E_{Z} \neq 0$ and $k_{B} T<\Gamma_{0}$ for nonmagnetic leads. For the ferromagnetic case the spin current can be seen even for $E_{Z}=0$ and high temperatures $\left(k_{B} T>\Gamma_{0}\right)$. In particular $I^{s}$ goes to zero as the time evolves in the AP alignment and remains finite in the stationary limit for the $\mathrm{P}$ configuration. The tunnel magnetoresistance was also analyzed. We found quantum coherent beats and negative values of the TMR due to the transient dynamics of incoming and outgoing spin polarized electrons in the quantum dot. The negative vales of the TMR vanish as the temperature increases, which is confirmed by master equation calculations.

The author acknowledges A. P. Jauho, J. C. Egues and J. P. Morten for valuable comments and suggestions. The author also acknowledges the kind hospitality at MICDTU (Denmark) during the final stages of this work. This work was supported by the Brazilian Ministry of Science and Technology and IBEM (Brazil).
1 G. A. Prinz, Science 282, 1660 (1998); S. A. Wolf, D. D. Awschalom, R. A. Buhrman, J. M. Daughton, S. von Molnár, M. L. Roukes, A. Y. Chtchelkanova, and D. M. Treger, Science 294, 1488 (2001); Semiconductor Spintronics and Quantum Computation, eds. D. D. Awschalom, D. Loss, and N. Samarth, Springer, Berlin (2002); D. D. Awschalom and M. E. Flatté, Nat. Phys. 3, 153 (2007).

2 S. Datta and B. Das, Appl. Phys. Lett. 56 (7), 665 (1990).

3 M. E. Flatté and G. Vignale, Appl. Phys. Lett. 78, 1273 (2001).

4 S. Cortez, O. Krebs, S. Laurent, M. Senes, X. Marie, P. Voisin, R. Ferreira, G. Bastard, J.-M. Gérard, and T. Amand, Phys. Rev. Lett. 89, 207401 (2002).

5 M. Kroutvar, Y. Ducommun, D. Heiss, M. Bichler, D. Schuh, G. Abstreiter, and J. J. Finley, Nature 432, 81 (2004).

6 D. P. DiVincenzo, Science 270, 255 (1995).

7 D. Loss and D. P. DiVincenzo, Phys. Rev. A 57, 120 (1998).

8 M. A. Nielsen and I. L. Chuang, Quantum Computation and Quantum Information, Cambridge Univ. Press, Cambridge (2000).

9 T. Hayashi, T. Fujisawa, H. D. Cheong, Y. H. Jeong, and Y. Hirayama, Phys. Rev. Lett. 91, 226804 (2003).

10 J. M. Elzerman, R. Hanson, L. H. W. van Beveren, B. Witkamp, L. M. K. Vandersypen, and L. P. Kouwenhoven, Nature 430, 431 (2004).

11 J. R. Petta, A. C. Johnson, J. M. Taylor, E. A. Laird, A. Yacoby, M. D. Lukin, C. M. Marcus, M. P. Hanson, A. C. Gossard, Science 309, 2180 (2005).
12 M. V. G. Dutt, J. Cheng, B. Li, X. Xu, X. Li, P. R. Berman, D. G. Steel, A. S. Bracker, D. Gammon, S. E. Economou, R.-B. Liu, and L. J. Sham, Phys. Rev. Lett. 94, 227403 (2005)

13 F. H. L. Koppens, C. Buizert, K. J. Tielrooij, I. T. Vink, K. C. Nowack, T. Meunier, L. P. Kouwenhoven, and L. M. K. Vandersypen, Nature 442, 766 (2006).

14 A. Greilich, R. Oulton, E. A. Zhukov, I. A. Yugova, D. R. Yakovlev, M. Bayer, A. Shabaev, Al. L. Efros, I. A. Merkulov, V. Stavarache, D. Reuter, and A. Wieck, Phys. Rev. Lett. 96, 227401 (2006).

15 M. V. G. Dutt, J. Cheng, Y. Wu, X. Xu, D. G. Steel, A. S. Bracker, D. Gammon, S. E. Economou, R.-B. Liu, and L. J. Sham, Phys. Rev. B 74, 125306 (2006).

16 M. H. Mikkelsen, J. Berezovsky, N. G. Stoltz, L. A. Coldren, and D. D. Awschalom, Nat. Phys. 3, 770 (2007).

17 H.-A. Engel and D. Loss, Phys. Rev. B 65, 195321 (2002).

18 S. A. Crooker, D. D. Awschalom, J. J. Baumberg, F. Flack, and N. Samarth, Phys. Rev. B 56, 7574 (1997).

19 J. A. Gupta, D. D. Awschalom, X. Peng, and A. P. Alivisatos, Phys. Rev. B 59, 10421(R) (1999).

20 J. M. Kikkawa and D. D. Awschalom, Science 287, 473 (2000).

21 J. C. Egues, C. Gould, G. Richter, and L. W. Molenkamp, Phys. Rev. B 64, 195319 (2001).

22 S. A. Gurvitz, D. Mozyrsky, and G. P. Berman, Phys. Rev. B 72, 205341 (2005).

23 N. S. Wingreen, A. P. Jauho, and Y. Meir, Phys. Rev. B 48, 8487 (1993).

24 A. P. Jauho, N. S. Wingreen, and Y. Meir, Phys. Rev. B 
50, 5528 (1994).

25 A. P. Jauho, N. S. Wingreen, and Y. Meir, Semicond. Sci. Technol. 9, 926 (1994).

26 J. Maciejko, J. Wang, and H. Guo, Phys. Rev. B 74, 085324 (2006).

27 For some values of the Zeeman energy in a semiconductor quantum dot see, for instance, R. Hanson, B. Witkamp, L. M. K. Vandersypen, L. H. W. Beveren, J. M. Elzerman, and L. P. Kouwenhoven, Phys. Rev. Lett. 91, 196802 (2003).

28 In principle this is experimentally possible by applying a local magnetic field or by using a lead's material with small enough $g$ factor in order to have $E_{Z}^{\text {leads }} \ll E_{F}$.

29 No Coulomb interaction is accounted for in our model. Previous results in the literature have shown that the cases of no electron repulsion in the $\operatorname{dot}(U=0)$ and high bias regime $\left(U \ll E_{F}-E_{d}\right)$ give similar results in the sequential-tunneling limit [see Ref. 39]. This however is not straightforward extended to the present coherent case $\left(k_{B} T \ll \Gamma_{0}\right)$. So further investigation on the effects of $U$ on the coherent time-dependent transport is desirable. Some results can already be seen, e.g., in Q. F. Sun, J. Wang, and T. H. Lin, Phys. Rev. B 58, 13007 (1998) and R. López, R. Aguado, G. Platero, and C. Tejedor, Phys. Rev. B 64, 075319 (2001).

30 A. V. Khaetskii and Y. V. Nazarov, Phys. Rev. B 61, 12639 (2000); ibid. 64, 125316 (2001).

31 A. C. Johnson, J. R. Petta, J. M. Taylor, A. Yacoby, M. D. Lukin, C. M. Marcus, M. P. Hanson, and A. C. Gossard, Nature 435, 925 (2005).

32 A. V. Khaetskii, D. Loss, and L. Glazman, Phys. Rev. Lett.
88, 186802 (2002).

${ }^{33}$ R. de Sousa and S. Das Sarma, Phys. Rev. B 67, 033301 (2003).

34 H. Haug and A. P. Jauho, Quantum Kinetics in Transport and Optics of Semiconductors, Springer Solid-State Sciences 123 (1996).

35 W. Rudziński and J. Barnaś, Phys. Rev. B 64, 85318 (2001).

36 This expression was originally derived in 23] for the spin independent case.

37 We follow the notation of H. Bahlouli, Phys. Stat. Sol. (a) 179, 475 (2000).

38 G. Kiesslich, A. Wacker, and E. Schöll, Phys. Rev. B 68, 125320 (2003).

39 F. M. Souza, S. A. Leão, R. M. Gester, and A. P. Jauho, Phys. Rev. B 76, 125318 (2007).

40 Here we present the current and the spin current in the emitter lead. The collector current and spin current show no significant contrasting features.

41 For some standard values of $\Gamma_{0}$ see, for instance, D. G.Gordon, J. Göres, M. A. Kastner, H. Shtrikman, D. Mahalu, and U. Meirav, Phys. Rev. Lett. 81, 5225 (1998); F. Simmel, R. H. Blick, J. P. Kotthaus, W. Wegscheider, and M. Bichler, ibid. 83, 804 (1999); D. G.-Gordon, H. Shtrikman, D. Mahalu, D. A.-Magder, U. Meirav, M. A. Kastner, Nature 391, 156 (1998).

42 The negative values of the TMR observed here are not physically equivalent to those seen in [39], where negative values are found for $k_{B} T \gg \Gamma_{0}$. 